\title{
Thromboembolism in the Sub-Acute Phase of Spinal Cord Injury: A Systematic Review of the Literature
}

\author{
Samer Alabed ${ }^{1,2}$, Maurizio Belci ${ }^{1}$, Joost J Van Middendorp ${ }^{1}$, Ahmed Al Halabi ${ }^{3}$,Tom M Meagher ${ }^{4}$ \\ ${ }^{1}$ National Spinal Injuries Centre, Stoke Mandeville Hospital, Aylesbury, UK \\ ${ }^{2}$ Academic Unit of Radiology, University of Sheffield, Sheffield, UK \\ ${ }^{3}$ Department of Vascular Surgery, General Hospital of Celle, Germany \\ ${ }^{4}$ Radiology Department, Stoke Mandeville Hospital, Aylesbury, UK
}

To review the evidence of thromboembolism incidence and prophylaxis in the sub-acute phase of spinal cord injury (SCI) 3-6 months post injury. All observational and experimental studies with any length of follow-up and no limitations on language or publication status published up to March 2015 were included. Two review authors independently selected trials for inclusion and extracted data. Outcomes studied were incidence of pulmonary embolism (PE) and deep vein thrombosis (DVT) in the sub-acute phase of SCl. The secondary outcome was type of thromboprophylaxis. Our search identified 4305 references and seven articles that met the inclusion criteria. Five papers reported PE events and three papers reported DVT events in the sub-acute phase of SCI. Studies were heterogeneous in populations, design and outcome reporting, therefore a meta-analysis was not performed. The included studies report a PE incidence of $0.5 \%-6.0 \%$ and DVT incidence of $2.0 \%-8.0 \%$ in the sub-acute phase of SCl. Thromboprophylaxis was poorly reported. Spinal patients continue to have a significant risk of PE and DVT after the acute period of their injury. Clinicians are advised to have a low threshold for suspecting venous thromboembolism in the sub-acute phase of $\mathrm{SCl}$ and to continue prophylactic anticoagulation therapy for a longer period of time.

Keywords: Spinal cord injury; Thromboembolism; Deep vein thrombosis; Pulmonary embolism; Rehabilitation; Systematic review

\section{Introduction}

Venous thromboembolism (VTE) in spinal cord injury (SCI) patients has limited clinical signs. Deep vein thrombosis (DVT) presents frequently with leg oedema and fever as the only features [1]. However, this can also be mimicked by heterotopic ossification or cellulitis [1]. Blood tests including D-dimer are of limited benefit $[2,3]$ and compression ultrasound is the current mainstay in the diagnosis for DVT. It is widely available, accurate and non-invasive [4]. Venography is the gold standard in diagnosing DVT, however is invasive, more expensive and involves contrast injection. It is not routinely used [4]. Pulmonary embolism (PE) may present with dyspnoea, tachycardia, syncope or chest pain. Neuropathic pain, intercostal muscle paralysis and respiratory tract infections can have similar presentations and this contributes to the difficulty in diagnosing PE [1]. Diagnostic algorithms including Wells and modified Geneva scoring systems have limited applicability in spinal patients $[5,6]$. Pulmonary

Received Feb 15, 2016; Revised Apr 2, 2016; Accepted May 3, 2016

Corresponding author: Samer Alabed

Academic Unit of Radiology, C floor, Royal Hallamshire Hospital, Glossop Road, Sheffield, S10 2JF, UK

Tel: +44-114-271-1643, Fax: +44-114-271-1714, E-mail: s.alabed@nhs.net 
angiography using multidetector computed tomography (CTPA) is the imaging modality of choice in diagnosing PE [7].

Spinal patients have the highest risk of thromboembolic events amongst all hospitalized patient groups [8-11]. While most studies have concentrated on the incidence of PE and DVT in the acute phase of SCI, research on the sub-acute and chronic rehabilitation phases are lacking [12]. Thromboprophylaxis is given in the acute phase based on observational evidence that PE is a significant cause of death in the first 90 days post injury $[10,13]$. There is no thromboprophylaxis recommended in the sub-acute rehabilitation phase [14]. Knowing the incidence of thromboembolic events in the sub-acute rehabilitation phase is important to estimate risk and facilitate evidence based prevention in this period of injury.

\section{Objective}

To review the evidence of thromboembolism incidence and prophylaxis in the sub-acute rehabilitation of SCI.

\section{Methods}

The protocol of this systematic review was registered in the international database of prospectively registered systematic reviews in health and social care (PROSPERO) on 29 August 2014 (registration number: CRD42014013471) [15].

\section{Eligibility Criteria for studies for this review}

1) Types of studies

All experimental and observational studies with any length of follow-up were included. No limitations on language or publication status were applied. Case reports were excluded.

\section{2) Types of participants}

We included studies of SCI at any level or mechanism of injury and of any age or gender. Exclusion criteria were studies with a follow-up finishing within the first 3 months of injury and studies that only included patients more than 6 months after injury.

\section{Types of outcome measures}

Studies were eligible if they reported thromboembolic events including DVTs or PEs in the sub-acute phase of SCI (3-6 months post injury).

\section{Outcomes}

The three outcomes were incidence of PE in the sub-acute phase of SCI, incidence of DVT in the sub-acute phase of SCI and thromboprophylaxis used in the sub-acute phase.

\section{Search methods for identification of studies}

The Cochrane Central Register of Controlled Trials (CENTRAL, March 2015), MEDLINE (HDAS, 1950 to March 2015) and EMBASE Classic and EMBASE (HDAS, 1980 to March 2015) databases were searched as summarized by the medical librarian at Stoke Mandeville Hospital. The search strategy was designed for maximum sensitivity using free text, thesaurus terms and exploded thesaurus terms. There were no language or time restrictions in the searches. Appendix 1 provides full details. Additionally, the references of relevant studies were screened for eligible papers.

\section{Data collection and analysis}

Two review authors (S.A., A.H.) independently reviewed the titles and abstracts identified from the search. Fulltext publications were obtained when necessary and eligibility was determined independently by the authors. The authors independently extracted data from the studies fulfilling the inclusion criteria and assessed quality of included studies. The data was extracted using an agreed data extraction form. Where the incidence of PE or DVT was not directly reported in the included studies, it was calculated from the results. We adopted the Cochrane risk of bias tool for non-randomized trials (ACROBAT-NRSI) to assess the quality of included studies [16]. The tool assesses domains of sequence generation, allocation concealment, confounding, outcome measurement and assessment and follow-up.

\section{Results}

A total of 4305 references were identified from the search. The search at the Cochrane Library, MEDLINE and EMBASE retrieved 145, 1,199, and 2,961 results, respectively. After exclusion of duplicate articles, a total of 2,770 references were left. After screening titles and abstracts, 
172 papers were judged relevant. The full text of these was retrieved, and four additional relevant articles were identified from cross-referencing. Of these, 165 articles reported VTE in the acute phase of SCI or did not document VTE timing. Seven studies reported PE in the subacute phase as part of mortality investigation or only reported post-mortem PE diagnosed by autopsy. Those studies were thought to be of limited benefit in estimating $\mathrm{PE}$ incidence in living patients and so were not relevant to this review. Seven articles met the inclusion criteria (Fig. 1). No randomized trials were identified. Included studies were retrospective case series or cohort studies. Characteristics of the included studies are summarized in Table 1.

\section{Quality assessment}

Risk of bias in the seven studies is summarized in Fig. 2. No randomized controlled or quasi randomized trials were found. None of the included studies pre-specified a list of potential VTE confounders in the sub-acute phase. None of the studies involved statistical modelling for confounding factors. We could not tell from the included reports if confounding factors, such as infection, surgical intervention or lack of thromboprophylaxis, contributed to VTE incidence. Diagnosis of PE was based on perfusion lung scan using ${ }^{99 \mathrm{~m}}$ TC macro-aggregated albumin

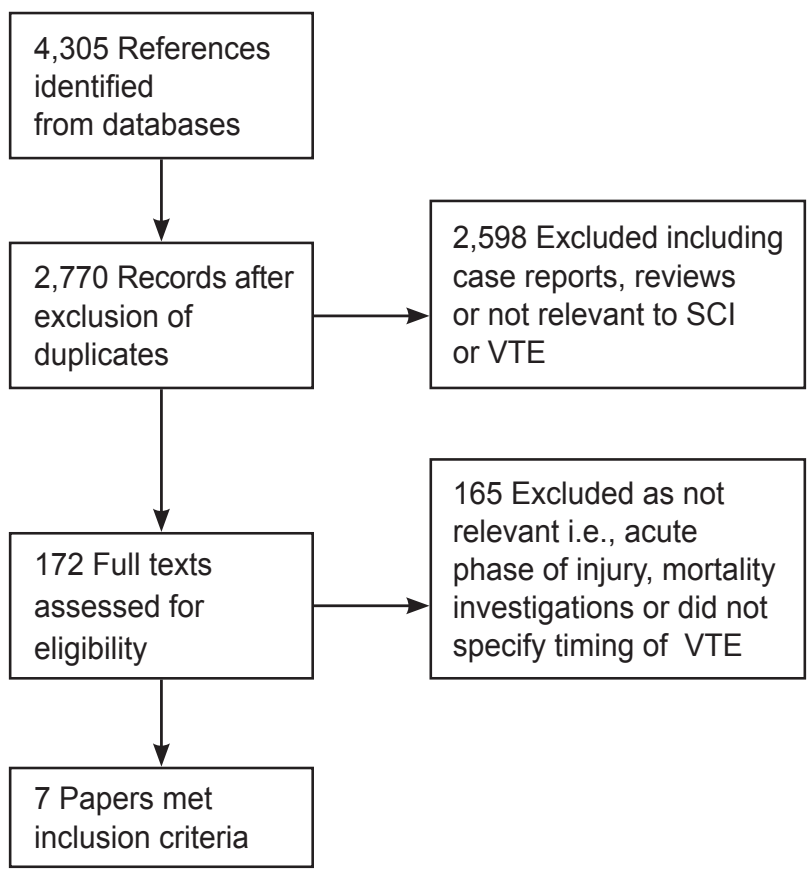

Fig. 1. Study flow diagram. SCI, spinal cord injury; VTE, venous thromboembolism. particle in one study [17] and using ventilation-perfusion scans and pulmonary angiography in another [18]. El Masri and Silver [19] diagnosed PEs clinically using the criterion of sudden onset of pleuritic chest pain lasting more than 12 hours with hemoptysis, after the exclusion of chest infection and a cardiac event. CTPA and perfusion lung scan were used to diagnose PE in one study [20]. We could not tell how PE diagnosed by Chung et al. [21]. DVT was diagnosed in McKinley et al. [18] clinically or with 1-125 labelled fibrinogen uptake citation, impedance plethysmography, Doppler or venography. In MackiewiczMile et al. [22] DVT was diagnosed by D-dimer and ultrasound Doppler. Lamb et al. [23] accepted any diagnostic method for VTE for inclusion; however we could not tell how patients were diagnosed with PE or DVT from the report. The study reported total VTE events without distinguishing between PE and DVT in the final results. Methods for collecting data were poorly reported and we

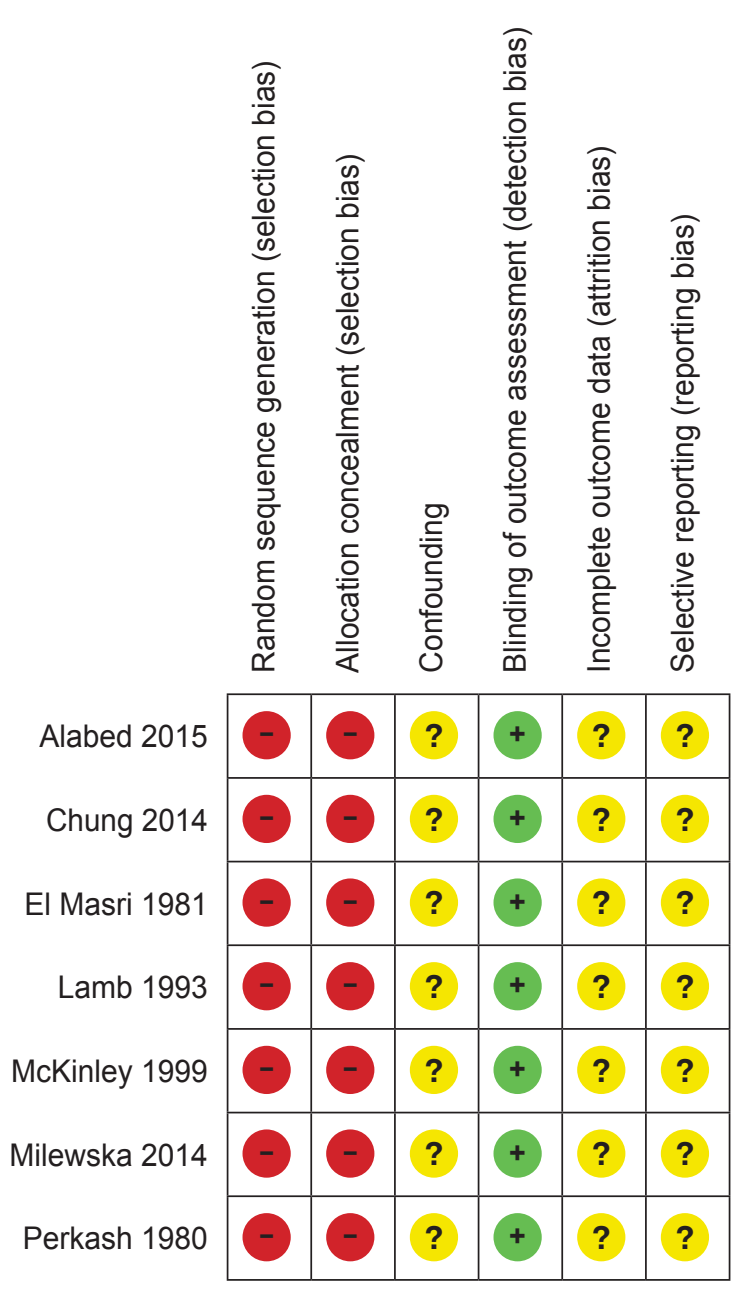

Fig. 2. Risk of bias summary. 


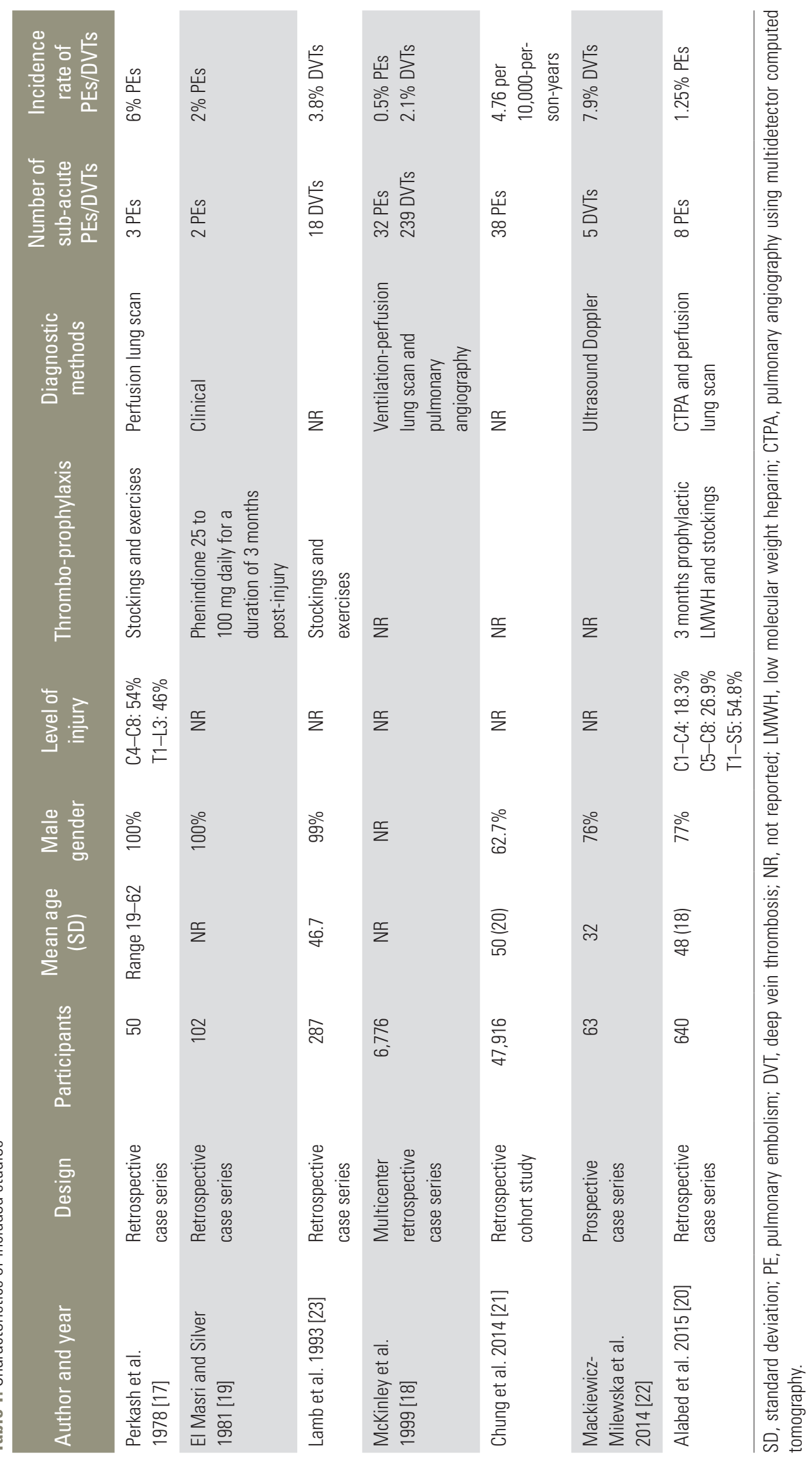


could not tell if any of the studies used more than one outcome assessor to repeat the analysis to ensure reliability. However, as PE and DVT were diagnosed using objective imaging methods the risk of bias in outcome assessment was thought to be low. In Alabed et al. [20] data about CTPA and perfusion scans were collected from Centricity Radiology Information Systems. Images were reported by different consultant radiologists. The included papers did not report incomplete or missing data. We could not tell if this was because of lack or reporting or absence of missing data.

\section{Pulmonary Embolism}

Five papers reported PEs in the sub-acute phase. We did not pool data in a meta-analysis because of differences in populations, study designs and outcome reporting in the included studies. We reported the incidence of PE as reported by the included papers. However, where the included papers did not report the incidence of PE, we calculated it, if possible, from the reported findings. Perkash et al. [17] is a retrospective case series that reviewed 50 spinal patients at the Veterans Administration Hospital, Palo Alto, California between 1976 and 1977. They reported a total of 4 PEs, of which 3 were in the sub-acute phase. Two PEs in the sub-acute phase were recurrent episodes of thromboembolism. All PEs were confirmed on perfusion lung scan. The calculated PE incidence is 6\%. El Masri and Silver [19] reported on a retrospective case series of 102 consecutive patients admitted to the National Spinal Injuries Centre, Aylsebury, UK between 1976 and 1979. They reported 19 PEs, of which two were in the sub-acute phase. Both patients with PE had inadequate anticoagulation (less than 6 weeks) and one had multiple PEs prior to the sub-acute PE. The calculated sub-acute PE incidence was $2 \%$. McKinley et al. [18] described a multicenter retrospective case series. The data of 6,772 spinal patients were reviewed from the National Spinal Cord Injury Statistical Centre, Birmingham, AL, from 1973 to 1998. The diagnosis of PE reported in the database was made clinically or confirmed with ventilation-perfusion scans or pulmonary angiography. The study reported a PE incidence of $0.5 \%$ within the first year of SCI. We could not tell the subacute $\mathrm{PE}$ incidence separately from the reported findings. Chung et al. [21] was a retrospective cohort study involving data retrieved from the National Health Insurance Research Database of Taiwan from 1998 to 2008 . The study included 47,916 spinal patients and 191,664 matched non-SCI controls. It reported a PE incidence of 4.76 per 10,000 -person-years in the sub-acute phase compared to 1.48 per 10,000-person-years in the non-spinal patient group. Alabed et al. [20] described a retrospective case series of 640 spinal patients at the aforementioned National Spinal Injuries Centre. The study reviewed every CTPA or perfusion lung scan for all new admissions between 2008 and 2013. The PE incidence in the sub-acute phase was $1.25 \%$; $95 \%$ confidence interval $0.39-2.11$.

\section{Deep venous thrombosis}

Three studies reported on DVTs in the sub-acute phase. Lamb et al. [23] reported a retrospective case series that reviewed the medical charts of 287 spinal patients treated at the Spinal Cord Injury Centre, Zablocki Veteran's Affairs Medical Centre, Milwaukee, WI. VTE was counted if it was mentioned in the patient's chart regardless of diagnostic method used. The study reported total VTE incidence without specifying if the event was a PE or DVT. Eleven patients had VTE events in the sub-acute phase. We could not tell if these events were DVTs or PEs or both. The VTE incidence was 5.9\% in the acute phase and $3.8 \%$ in the sub-acute phase. McKinley et al. [18] reported a DVT incidence rate of $2.1 \%$ in the first year postinjury. We could not tell the sub-acute DVT incidence separately from the reported findings. In the third study, Mackiewicz-Milewska et al. [22] presented an abstract of a prospective case series that included 63 spinal patients from the rehabilitation department in Bydgoszcz, Poland. The study reported five DVTs in the sub-acute phase diagnosed with Doppler ultrasound. The DVT incidence in the sub-acute phase was $7.9 \%$.

\section{Thromboprophylaxis}

The included studies poorly reported type, dose or duration of anticoagulation used. In the study of El Masri and Silver [19], 66 of 102 patients received prophylactic anticoagulation with phenindione 25 to $100 \mathrm{mg}$ daily for up to 3 months post-injury, and the remaining 36 patients were either admitted after 3 months post injury or had contraindications to anticoagulation. No patient diagnosed with PE was adequately anti-coagulated at the time of PE due to contraindications or interventions. Lamb et al. [23] did not use prophylactic anticoagulation. Prophy- 
lactic compression stockings and leg exercise were used. Perkash et al. [17] reported the use of prophylactic compression stockings and leg exercise. We could not tell if prophylactic anticoagulation was used or not. In Alabed et al. [20], all patients received prophylactic anticoagulation with injections of low molecular weight heparin and use of compression stockings for up to 3 months post injury. We could not tell what type of thromboprophylaxis was used in McKinley et al. [18] or Chung et al. [21]. No study reported thromboprophylaxis beyond 90 days post SCI.

\section{Discussion}

Seven studies reported PE and DVT events in the subacute phase of SCI. The included studies were retrospective case series or cohort studies. The included studies reported a PE incidence of $0.5 \%-6.0 \%$ and a DVT incidence of $2.0 \%-8.0 \%$ in the sub-acute phase of SCI. This is lower than the $6 \%-12 \%$ incidence in the acute phase of SCI $[3,24]$. However, the incidence of PE in the sub-acute phase of SCI remains significantly higher than that of the normal population $(0.06 \%$ /year $)[25,26]$.

Thromboprophylaxis methods used were poorly documented. It is not clear whether VTEs occurred due to absence of thromboprophylaxis, lack of compliance or if VTEs occurred despite thromboprophylaxis. Current practice is to give 3 months of anticoagulation post-injury. However, as the risk of VTE remains significant up to 6 months post-injury, there is a need to investigate the efficacy of extending thromboprophylaxis to cover the subacute injury period. Clinicians are advised to have a low threshold for suspecting VTE in the sub-acute phase of SCI.

\section{Study Limitations}

Studies were retrospective without a clear follow-up period. Variations between studies did not permit a metaanalysis to calculate an incidence rate for PE or DVT. Only one included study used CTPA to diagnose PE [20]. As CTPA is the current gold standard for diagnosing PE, incidence of PE might be higher than reported in the included studies.

Two studies reported PE and DVT up to 12 months post-injury $[18,21]$. Both studies were included as they provided information about VTE events during the period of interest. However, as they covered a period longer then the sub-acute phase, they may not accurately reflect the incidence of VTE in the sub-acute phase.

\section{Conclusions}

This review includes papers reporting PE and DVT events in the sub-acute phase of SCI when thromboprophylaxis is routinely stopped. This review highlights the fact that spinal patients continue to have a significant risk of PE and DVT after thromboprophylaxis is stopped in the subacute phase of the injury. More evidence in the form of larger, well documented prospective cohort studies with follow-up is needed to determine exact VTE incidence rates.

\section{What is already known on this topic}

The risk of VTE in acute spinal cord injury is one of the highest among patients' groups. Current guidelines recommend 3 months thromboprophylaxis post injury.

\section{What this study adds}

There remains a significant risk of VTE in the sub-acute spinal injury period. However, a large prospective cohort studies is needed to establish the real risk of thromboembolism in this period. Double-blind randomized controlled trials are needed to establish the effect of extending thromboprophylaxis to cover the sub-acute injury period.

\section{Conflict of Interest}

No potential conflict of interest relevant to this article was reported.

\section{Acknowledgments}

We would like to thank Lorena Cascant and Phil Ruston the medical librarians at Stoke Mandeville Hospital for their precious help with the literature searches and retrieving the full texts of studies.

\section{References}

1. Saraf SK, Rana RJ, Sharma OP. Venous thromboembolism in acute spinal cord injury patients. Indian J Orthop 2007;41:194-7. 
2. Lee JS, Kim YT. Value of the D-dimer test in diagnosing venous thromboembolism in acute rehabilitation inpatients in Korea. PM R 2010;2(9 Suppl):S155.

3. Riklin C, Baumberger M, Wick L, Michel D, Sauter $B$, Knecht H. Deep vein thrombosis and heterotopic ossification in spinal cord injury: a 3 year experience at the Swiss Paraplegic Centre Nottwil. Spinal Cord 2003;41:192-8.

4. Teasell RW, Hsieh JT, Aubut JA, et al. Venous thromboembolism after spinal cord injury. Arch Phys Med Rehabil 2009;90:232-45.

5. Klok FA, Mos IC, Nijkeuter M, et al. Simplification of the revised Geneva score for assessing clinical probability of pulmonary embolism. Arch Intern Med 2008;168:2131-6.

6. Douma RA, Gibson NS, Gerdes VE, et al. Validity and clinical utility of the simplified Wells rule for assessing clinical probability for the exclusion of pulmonary embolism. Thromb Haemost 2009;101:197-200.

7. Stein PD, Fowler SE, Goodman LR, et al. Multidetector computed tomography for acute pulmonary embolism. N Engl J Med 2006;354:2317-27.

8. Todd JW, Frisbie JH, Rossier AB, et al. Deep venous thrombosis in acute spinal cord injury: a comparison of 125I fibrinogen leg scanning, impedance plethysmography and venography. Paraplegia 1976;14:50-7.

9. Rossi EC, Green D, Rosen JS, Spies SM, Jao JS. Sequential changes in factor VIII and platelets preceding deep vein thrombosis in patients with spinal cord injury. Br J Haematol 1980;45:143-51.

10. Silver JR, Noori Z. Pulmonary embolism following anticoagulation therapy. Int Disabil Stud 1991;13:16-9.

11. Geerts WH. Prevention of venous thromboembolism in high-risk patients. Hematology Am Soc Hematol Educ Program 2006:462-6.

12. Frisbie JH, Sharma GV. The prevalence of pulmonary embolism in chronically paralyzed subjects: a review of available evidence. Spinal Cord 2012;50:400-3.

13. Walsh JJ, Tribe C. Phlebo-thrombosis and pulmonary embolism in paraplegia. Paraplegia 1965;3:209-13.

14. Kim SW, Charallel JT, Park KW, et al. Prevalence of deep venous thrombosis in patients with chronic spinal cord injury. Arch Phys Med Rehabil 1994;75:965-8.

15. Alabed S, Al Halabi A, Belci M, Van Middendorp J, Meagher T. Systematic review of thromboembolism in sub-acute phase of spinal cord injury [Internet]. York, UK: PROSPERO Int.; 2015 [cited 2016 Sep 20].
Available from: http://www.crd.york.ac.uk/prospero/ display_record.asp?ID=CRD42014013471.

16. Sterne J, Higgins J, Reeves B. A Cochrane risk of bias assessment tool: for non-randomized studies of interventions (ACROBAT-NRSI) [Internet]. [cited 2015 Jul 1]. Available from: http://www.riskofbias.info.

17. Perkash A, Prakash V, Perkash I. Experience with the management of thromboembolism in patients with spinal cord injury: Part I. Incidence, diagnosis and role of some risk factors. Paraplegia 1978;16:322-31.

18. McKinley WO, Jackson AB, Cardenas DD, DeVivo MJ. Long-term medical complications after traumatic spinal cord injury: a regional model systems analysis. Arch Phys Med Rehabil 1999;80:1402-10.

19. El Masri WS, Silver JR. Prophylactic anticoagulant therapy in patients with spinal cord injury. Paraplegia 1981;19:334-42.

20. Alabed S, de Heredia LL, Naidoo A, Belci M, Hughes RJ, Meagher TM. Incidence of pulmonary embolism after the first 3 months of spinal cord injury. Spinal Cord 2015;53:835-7.

21. Chung WS, Lin CL, Chang SN, Chung HA, Sung FC, $\mathrm{Kao} \mathrm{CH}$. Increased risk of deep vein thrombosis and pulmonary thromboembolism in patients with spinal cord injury: a nationwide cohort prospective study. Thromb Res 2014;133:579-84.

22. Mackiewicz-Milewska M, Jung S, Kroszczynski AC, et al. Deep venous thrombosis in patients with chronic spinal cord injury. J Spinal Cord Med 2016;39:400-4.

23. Lamb GC, Tomski MA, Kaufman J, Maiman DJ. Is chronic spinal cord injury associated with increased risk of venous thromboembolism? J Am Paraplegia Soc 1993;16:153-6.

24. Haisma JA, van der Woude LH, Stam HJ, et al. Complications following spinal cord injury: occurrence and risk factors in a longitudinal study during and after inpatient rehabilitation. J Rehabil Med 2007; 39:393-8.

25. Oger E. Incidence of venous thromboembolism: a community-based study in Western France. EPIGETBP Study Group. Groupe d'Etude de la Thrombose de Bretagne Occidentale. Thromb Haemost 2000;83:657-60.

26. Spencer FA, Emery C, Lessard D, et al. The Worcester Venous Thromboembolism study: a populationbased study of the clinical epidemiology of venous thromboembolism. J Gen Intern Med 2006;21:722-7. 


\section{Appendix 1.}

\section{Search Strategies}

MEDLINE database was searched via HDAS platform from 1950 to March 18th 2015 for relevant studies.

The search strategy has:

- Used both free text and thesaurus terms

- Exploded thesaurus terms to capture narrower terms

- Used the adjacency operator (adj)

- Not used subheadings as are rarely used at systematic reviews

- Not restricted to major focus as is rarely used at systematic reviews

\begin{tabular}{|c|c|}
\hline 1. MEDLINE & exp SPINAL CORD INJURIES/ \\
\hline 2. MEDLINE & exp SPINAL FRACTURES/ \\
\hline 3. MEDLINE & exp PARAPLEGIA/ \\
\hline 4. MEDLINE & exp QUADRIPLEGIA/ \\
\hline 5. MEDLINE & (paraplegi* OR paraparesis).ti,ab \\
\hline 6. MEDLINE & (quadriplegi* OR quadriparesi* ${ }^{*}$ ).ti,ab \\
\hline 7. MEDLINE & (tetraplegia* ${ }^{*}$ OR tetraplagi* ${ }^{*}$ OR tetraparesis).ti,ab \\
\hline 8. MEDLINE & $\begin{array}{l}\left(\left(\text { spine OR spinal) adj3 (broken OR break }{ }^{*} \text { OR fracture* } \text { OR wound }^{*} \text { OR trauma* } \text { OR injur }^{*}\right.\right. \\
\text { OR damag* }) \text { ).ti,ab }\end{array}$ \\
\hline 9. MEDLINE & ((spinal cord) adj3 (contusion OR laceration OR trauma OR injur ${ }^{\star}$ OR ischemi $\left.\left.{ }^{\star}\right)\right) . t i, a b$ \\
\hline 10. MEDLINE & “central cord injury syndrome”.ti,ab \\
\hline 11. MEDLINE & (myelopathy adj3 (traumatic OR post-traumatic)).ti,ab \\
\hline 12. MEDLINE & exp CENTRAL CORD SYNDROME/ \\
\hline 13. MEDLINE & exp SPINAL CORD ISCHEMIA/ \\
\hline 14. MEDLINE & 1 OR 2 OR 3 OR 4 OR 5 OR 6 OR 7 OR 8 OR 9 OR 10 OR 11 OR 12 OR 13 \\
\hline 15. MEDLINE & THROMBOSIS/ \\
\hline 16. MEDLINE & THROMBOEMBOLISM/ \\
\hline 17. MEDLINE & exp VENOUS THROMBOSIS/ \\
\hline 18. MEDLINE & exp PULMONARY EMBOLISM/ \\
\hline 19. MEDLINE & EMBOLISM/ \\
\hline 20. MEDLINE & VENOUS THROMBOEMBOLISM/ \\
\hline 21. MEDLINE & (pulmonary adj2 (embolism OR thromboembolism)).ti,ab \\
\hline 22. MEDLINE & ((venous OR “deep venous" OR “deep-venous" OR “deep vein” OR “deep-vein”) adj2 thrombos”).ti,ab \\
\hline 23. MEDLINE & ((PE OR DVT OR VTE)).ti,ab \\
\hline 24. MEDLINE & (venous adj3 thromboemboli*).ti,ab \\
\hline 25. MEDLINE & 15 OR 16 OR 17 OR 18 OR 19 OR 20 OR 21 OR 22 OR 23 OR 24 \\
\hline 26. MEDLINE & 14 AND 25 \\
\hline
\end{tabular}


EMBASE database was searched via HDAS platform from 1980 to 18th March 2015 for relevant studies.

The search strategy has:

- Used both free text and thesaurus terms

- Exploded thesaurus terms to capture narrower terms

- Used the adjacency operator (adj)

- Not used subheadings as are rarely used at systematic reviews

- Not restricted to major focus as is rarely used at systematic reviews

\begin{tabular}{|c|c|}
\hline 27. EMBASE & exp SPINAL CORD INJURY/ \\
\hline 28. EMBASE & exp SPINE FRACTURE/ \\
\hline 29. EMBASE & exp PARAPLEGIA/ \\
\hline 30. EMBASE & exp QUADRIPLEGIA/ \\
\hline 31. EMBASE & (paraplegi* OR paraparesis).ti,ab \\
\hline 32. EMBASE & (quadriplegi ${ }^{*}$ OR quadriparesi ${ }^{*}$ ).ti,ab \\
\hline 33. EMBASE & (tetraplegia* OR tetraplagi* OR tetraparesis).ti,ab \\
\hline 34. EMBASE & $\begin{array}{l}\left(\left(\text { spine OR spinal) adj3 (broken OR break }^{*} \text { OR fracture* } \text { OR wound }^{*} \text { OR trauma* OR injur }\right.\right. \\
\left.\text { OR damag* }{ }^{*}\right) \text {.ti,ab }\end{array}$ \\
\hline 35. EMBASE & $\left(\left(\right.\right.$ spinal cord) adj3 (contusion OR laceration OR trauma OR injur* OR ischemi $\left.\left.{ }^{*}\right)\right)$.ti,ab \\
\hline 36. EMBASE & “central cord injury syndrome”.ti,ab \\
\hline 37. EMBASE & (myelopathy adj3 (traumatic OR post-traumatic)).ti,ab \\
\hline 38. EMBASE & exp CENTRAL CORD SYNDROME/ \\
\hline 39. EMBASE & exp SPINAL CORD ISCHEMIA/ \\
\hline 40. EMBASE & 27 OR 28 OR 29 OR 30 OR 31 OR 32 OR 33 OR 34 OR 35 OR 36 OR 37 OR 38 OR 39 \\
\hline 41. EMBASE & THROMBOSIS/ \\
\hline 42. EMBASE & THROMBOEMBOLISM/ \\
\hline 43. EMBASE & exp VEIN THROMBOSIS/ \\
\hline 44. EMBASE & exp LUNG EMBOLISM/ \\
\hline 45. EMBASE & EMBOLISM/ \\
\hline 46. EMBASE & VENOUS THROMBOEMBOLISM/ \\
\hline 47. EMBASE & $\left(\right.$ pulmonary adj2 $\left(\right.$ embolism $^{*}$ OR thromboembolism $\left.\left.{ }^{*}\right)\right) . t i, a b$ \\
\hline 48. EMBASE & ((venous OR “deep venous" OR “deep-venous" OR “deep vein” OR “deep-vein”) adj2 thrombos*).ti,ab \\
\hline 49. EMBASE & ((PE OR DVT OR VTE)).ti,ab \\
\hline 50. EMBASE & (venous adj3 thromboemboli*).ti,ab \\
\hline 51. EMBASE & 41 OR 42 OR 43 OR 44 OR 45 OR 46 OR 47 OR 48 OR 49 OR 50 \\
\hline 52. EMBASE & 40 AND 51 \\
\hline
\end{tabular}


The 6 Cochrane Library databases were searched for relevant studies from the below periods:

- Cochrane Database of Systematic Reviews (CDSR) from 2005 to March 18th 2015

- Database of Abstracts of Reviews of Effects (DARE) from 1994 to March 18th 2015

- Cochrane Central Register of Controlled Trials (CENTRAL) from 1898 to March 18th 2015

- Cochrane Methodology Register from 1951 to March 18th 2015

- Health Technology Assessment Database from 1989 to March 18th 2015

- NHS Economic Evaluation Database from 1968 to March 18th 2015

\begin{tabular}{|c|c|}
\hline$\# 1$ & MeSH descriptor: [Spinal Cord Injuries] explode all trees \\
\hline$\# 2$ & MeSH descriptor: [Spinal Fractures] explode all trees \\
\hline$\# 3$ & MeSH descriptor: [Paraplegia] explode all trees \\
\hline$\# 4$ & MeSH descriptor: [Quadriplegia] explode all trees \\
\hline$\# 5$ & paraplegi* $^{*}$ or paraparesis \\
\hline$\# 6$ & quadriplegi* $^{*}$ quadriparesi* \\
\hline$\# 7$ & tetraplegia* ${ }^{*}$ tetraplagi* or tetraparesis \\
\hline$\# 8$ & (spine or spinal) near $/ 3$ (broken or break ${ }^{*}$ or fracture ${ }^{*}$ or wound ${ }^{*}$ or trauma ${ }^{*}$ or injur ${ }^{*}$ or damag ${ }^{*}$ ) \\
\hline$\# 9$ & (spinal cord) near/3 (contusion or laceration or trauma or injur* or ischemi*) \\
\hline$\# 10$ & "central cord injury syndrome" \\
\hline$\# 11$ & myelopathy near/3 (traumatic or post traumatic) \\
\hline$\# 12$ & MeSH descriptor: [Central Cord Syndrome] explode all trees \\
\hline \#13 & MeSH descriptor: [Spinal Cord Ischemia] explode all trees \\
\hline$\# 14$ & $\# 1$ or $\# 2$ or $\# 3$ or $\# 4$ or $\# 5$ or $\# 6$ or $\# 7$ or $\# 8$ or $\# 9$ or $\# 10$ or $\# 11$ or $\# 12$ or $\# 13$ \\
\hline$\# 15$ & MeSH descriptor: [Thrombosis] this term only \\
\hline \#16 & MeSH descriptor: [Thromboembolism] this term only \\
\hline \#17 & MeSH descriptor: [Venous Thrombosis] explode all trees \\
\hline \#18 & MeSH descriptor: [Pulmonary Embolism] explode all trees \\
\hline \#19 & MeSH descriptor: [Embolism] this term only \\
\hline$\# 20$ & MeSH descriptor: [Venous Thromboembolism] this term only \\
\hline \#21 & pulmonary near/2 (embolism* or thromboembolism* ${ }^{*}$ \\
\hline \#22 & (venous or "deep venous" or "deep venous" or "deep vein" or "deep vein") near/2 thrombos* \\
\hline \#23 & PE or DVT or VTE \\
\hline$\# 24$ & venous near/3 thromboemboli* \\
\hline$\# 25$ & $\# 15$ or $\# 16$ or \#17 or \#18 or \#19 or \#20 or \#21 or \#22 or \#23 or \#24 \\
\hline \#26 & \#14 and \#25 \\
\hline
\end{tabular}

